\title{
Capnocytophagia Sepsis and Spontaneous Splenic Rupture after a Minor Dog Bite
}

\author{
Anthony R. Cyr, Brian S. Zuckerbraun, Juan Carlos Puyana, and Louis H. Alarcon
}

\begin{abstract}
Background: Spontaneous splenic rupture in critically ill patients without prior identified trauma or underlying splenic pathology is an uncommon event with multiple predisposing factors, including infectious etiologies. Case Presentation: We present the case of a 62-year-old female with history of mild chronic obstructive pulmonary disease and no prior known immunodeficiency who developed severe sepsis with multi-system organ failure two days after a mild dog bite on her wrist, with blood cultures positive for Capnocytophagia spp. She required anti-coagulation with heparin for disseminated intravascular coagulopathy (DIC) leading to deep vein thrombosis. Although she demonstrated resolution of sepsis, on hospital day 19 the patient experienced new onset of left upper quadrant pain; workup demonstrated spontaneous splenic rupture that required emergent splenectomy. She did well thereafter and continues to progress through inpatient rehabilitation from her sepsis. Conclusion: Capnocytophagia sepsis, although rare, progresses quickly to multi-system organ failure. Rapid identification of spontaneous splenic rupture may be challenging in these patients given the spectrum of their other physiologic abnormalities but is essential for appropriate surgical therapy.
\end{abstract}

$\mathbf{S}$ PONTANEOUS SPLENIC RUPTURE can be defined as rupture of the spleen in the absence of a sufficient traumatic event to produce injury. In the majority of these atraumatic splenic ruptures, there is underlying pathology such as neoplasia or undiagnosed inflammatory disease that predisposes patients to splenic rupture [1]. Various infectious processes may produce similar effects, with the classic examples being malaria and infectious mononucleosis. Here, we present the case of a 62-year-old immunocompetent female who developed severe Capnocytophagia sepsis after a dog bite and who developed rapidly multi-system organ failure including disseminated intravascular coagulation (DIC) and deep venous thrombosis (DVT). While recovering from the sequelae of this devastating illness she suffered spontaneous splenic rupture that required emergent splenectomy without any evidence of prior underlying splenic pathology.

\section{Case Presentation}

A 62-year-old female presented to her local emergency department with flu-like symptoms, myalgias, chills, watery diarrhea, and numbness and tingling in her distal extremities two days after suffering a minor dog bite on her hand. Her past medical history was remarkable for chronic obstructive pulmonary disease without home oxygen requirement, with a distant history of a provoked pulmonary embolism that was treated with six months of oral anti-coagulation. Her surgical history was substantial for hysterectomy and kidney stones treated with lithotripsy. In the emergency department, she was found to be tachycardic and hypotensive, and blood cultures were drawn. She did not respond to a fluid challenge with $1 \mathrm{~L}$ normal saline and was started on norepinephrine and emergently transferred to a tertiary medical center for further workup. On arrival, she remained hypotensive and initial laboratory values revealed a creatinine of 4.2 with profound thrombocytopenia of 18,000 and international normalized ratio (INR) of 2.9 without any concurrent pharmacologic anticoagulation. Non-contrast computed tomography (CT) scan of the chest, abdomen, and pelvis at the time of presentation revealed no obvious source for her clinical presentation and demonstrated normal splenic anatomy (Fig. 1A). Her initial physical examination was remarkable for developing purpura in her distal extremities and the tip of her nose, with a minor bite wound to her hand without evidence of substantial abscess

Division of Trauma and General Surgery, Department of Surgery, University of Pittsburgh, Pittsburgh, Pennsylvania.

(C) Anthony R. Cyr et al. 2016; Published by Mary Ann Liebert, Inc. This Open Access article is distributed under the terms of the Creative Commons License (http://creativecommons.org/licenses/by/4.0), which permits unrestricted use, distribution, and reproduction in any medium, provided the original work is properly credited. 

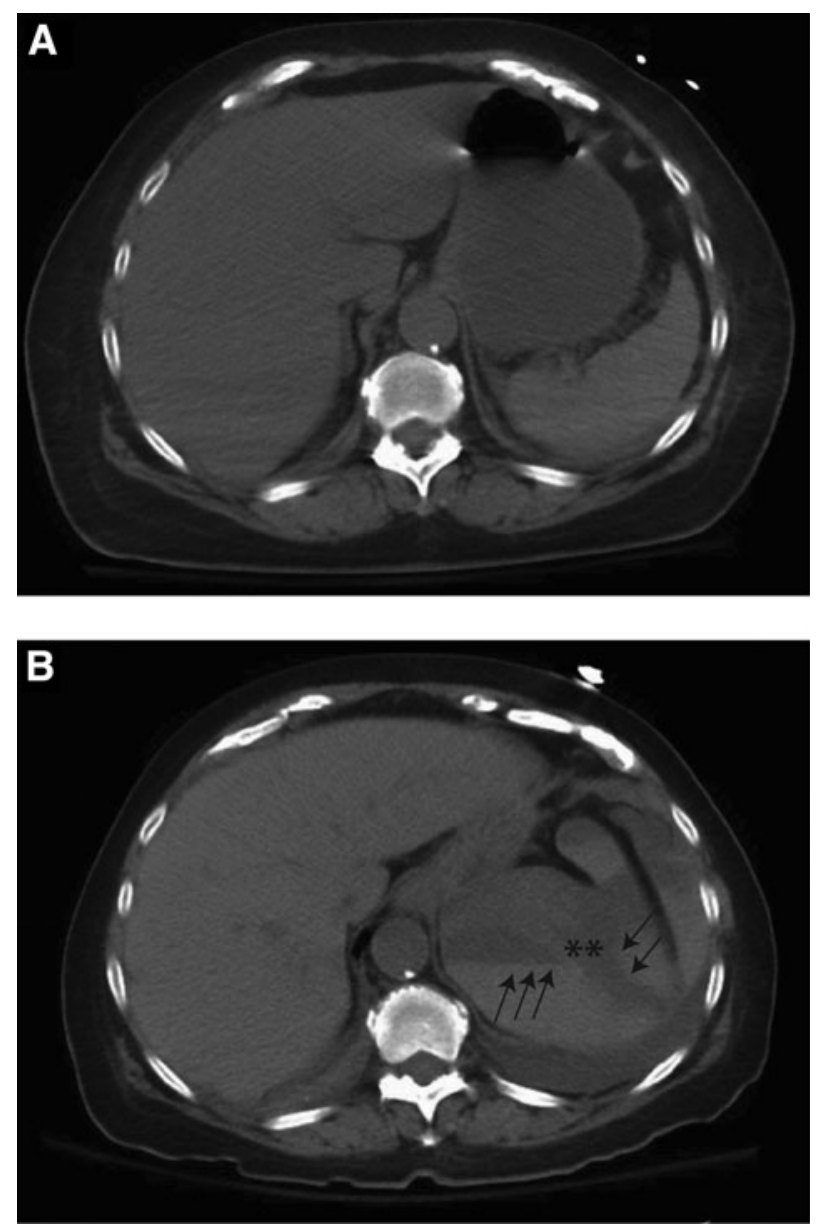

FIG. 1. (A) Computed tomography (CT) image demonstrating normal splenic anatomy on admission to the hospital. (B) Repeat CT scan demonstrating fractured splenic parenchyma (borders marked by arrowheads) and layering (marked by $*)$, concerning for spontaneous splenic rupture.

or cellulitis. Within $48 \mathrm{~h}$ she had developed florid multi-system organ failure. This included DIC, with multiple DVTs for which she was started on a heparin drip. She became anuric and required continuous renal replacement therapy. Her mental status deteriorated and she required intubation for airway protection. Her fingers, toes, and nose developed distal necrosis, and she suffered a substantial epistaxis event requiring Gelfoam ${ }^{\circledR}$ packing (Pfizer, New York, NY).

Multiple sets of blood cultures taken at our facility demonstrated no growth. The initial cultures taken from the referring hospital ultimately grew Capnocytophagia, and although individual speciation was not possible with the available laboratory equipment, the suspected pathogen was Capnocytophagia canimorsus. She was maintained on piperacillin-tazobactam therapy for empiric coverage, and over the next several days began improving. She was transitioned from continuous renal replacement to intermittent dialysis, was successfully extubated, and demonstrated some recovery of her cognitive function. She continued to experience tachycardia in the low 100s at baseline.

On hospital day 19 from initial presentation routine morning laboratory tests demonstrated a precipitous decrease in her hemoglobin to $4.8 \mathrm{mg} / \mathrm{dL}$ from $7.2 \mathrm{mg} / \mathrm{dL}$ the previous day. The patient remained tachycardic in the low 100s and systolic blood pressures were in the 90s. An unenhanced CT scan of the abdomen and pelvis demonstrated a shattered spleen with peri-splenic blood and layering concerning for active acute bleeding (Fig. 1B). On physical examination, the patient's mental status had not changed from her baseline and she continued to respond appropriately to questions. She did not have any abdominal pain or peritoneal signs and had no additional physical findings to accompany her concerning laboratory values and CT findings. Acute care surgery was consulted and the patient was taken to the operating room for emergency exploratory laparotomy. On entry to the abdomen approximately $600 \mathrm{~mL}$ of hematoma was evacuated from the left upper quadrant. A pulverized spleen was removed piecemeal with meticulous sequential hemostasis (Fig. 2). Formal control of the splenic hilum was not possible because of the complete obliteration of the normal splenic anatomy. The patient received a total of six units of packed red cells, two units of cryoprecipitate, and a six-pack of platelets as part of her resuscitation. She tolerated the procedure well. Pathology demonstrated extensive coagulative necrosis of the splenic parenchmya with substantial clot burden and minimal residual viable splenic tissue, without
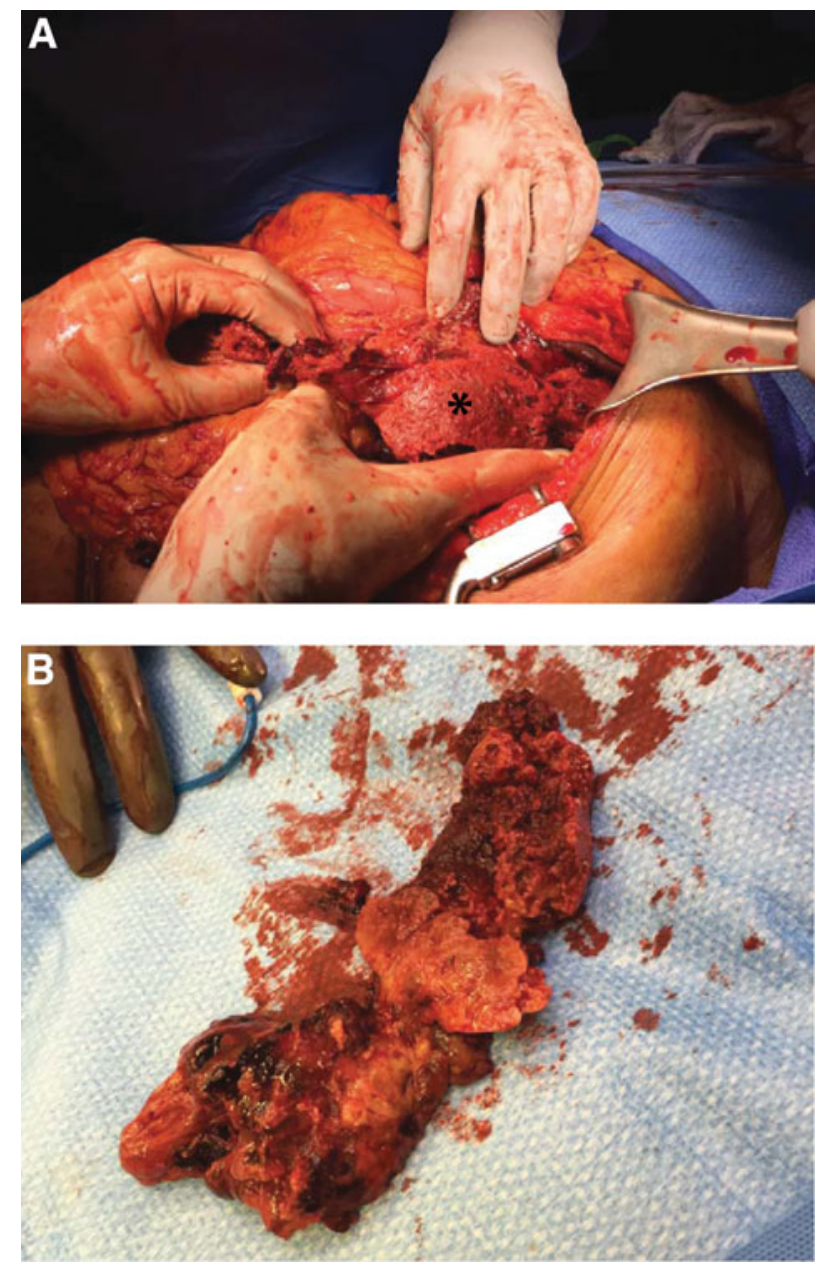

FIG. 2. (A) In situ demonstration of pulverized splenic parenchmya intra-operatively (spleen marked by *). (B) Ex vivo specimen demonstrating near-total disruption of normal anatomy and substantial damage to splenic parenchyma. 
evidence of underlying malignant disease or other condition. She received appropriate post-splenectomy vaccinations.

Several weeks post-operatively, while on therapeutic anticoagulation, she developed a retroperitoneal bleed not associated with the operative site. Pharmacolgic anti-coagulation was held and an inferior vena cava filter was placed. The remainder of her hospital course was dominated by rehabilitation efforts, and four months after the event she continues her recovery at home.

\section{Discussion}

Spontaneous splenic rupture, in the absence of trauma and prior underlying splenic parenchymal defect, is a rare but potentially deadly entity that requires urgent surgical intervention. This differs somewhat from the grading and management of traumatic splenic injuries, in which the otherwise healthy spleen is assumed to be capable of recovery from minor insults in the absence of hemodynamic instability. Moreover, the use of interventional radiology for embolization has also enabled the non-operative management of traumatic splenic injury, up to and including high-grade blunt splenic injury $[2,3]$. In spontaneous splenic injuries, without any identifiable trauma, surgery remains the mainstay of therapy because of the presumed underlying pathologic defect. Frequently, this is an underlying and potentially undiagnosed malignant disease, although other inflammatory disorders, pharmacologic agents, and infections may predispose individual patients to spontaneous splenic rupture [1]. In the United States, infectious mononucleosis is one of the most common infectious causes of spontaneous splenic rupture, with an estimated incidence of $0.1 \%$ among patients with infectious mononucleosis [4]. In contrast, malaria is a more common infectious cause of spontaneous splenic infarction and rupture worldwide, and its true incidence is likely under-reported in the literature [5]. Regardless of the associated causes, the critical management step in all cases of spontaneous splenic rupture is early identification to facilitate appropriate surgical management.

Here, we present a patient who presented with Capnocytophagia sepsis with florid multi-system organ failure after a dog bite, who progressed to atraumatic splenic rupture. Capnocytophagia sepsis is a rare but devastating complication of animal bites, with more than 160 case reports throughout the literature since the first description in 1976 [6,7]. Generally, although patients who are previously immunosuppressed and particularly those who are functionally asplenic are more susceptible to severe infection, no distinct comorbid condition is identified in as many as $40 \%$ of cases [6]. The diagnosis of Capnocytophagia can be challenging because of the fastidious nature of the bacterium, which frequently requires up to two weeks of incubation for appropriate colony analysis. Subsequently, early recognition of possible Capnocytophagia from clinical features in the setting of recent animal bites is critical for appropriate therapy of affected patients [7,8].

In our patient's case, we suspect two underlying risk factors contributed to her splenic rupture-global coagulation dysfunction with DIC as identified on presentation may have caused microvascular damage to the spleen itself, whereas subsequent persistent therapeutic anti-coagulation may have promoted ongoing slow intra-capsular bleeding that ultimately caused a splenic rupture. To our knowledge, there is no other reported case of splenic rupture in the setting of Capnocytophagia sepsis, although one report detailed total splenic thrombosis in the setting of Capnocytophagiaassociated thrombotic thrombocytopenic purpura [9].

\section{Conclusion}

Spontaneous splenic rupture is an uncommon clinical event that is generally associated with underlying splenic parenchymal pathology. Capnocytophagia infections after animal bites can cause severe sepsis with associated multisystem organ failure that likely predisposes affected patients to splenic injury based on both the initial insult to the coagulation cascade and the therapeutic interventions required thereafter. The presented case illustrates the substantial morbidity associated with Capnocytophagia sepsis as well as the importance of early recognition of spontaneous splenic rupture in patients who are critically ill.

\section{Author Disclosure Statement}

There are no conflicts of interest to report for any of the listed authors.

\section{References}

1. Renzulli P, Hostettler A, Schoepfer AM, et al. Systematic review of atraumatic splenic rupture. Br J Surg 2009;96: 1114-1121.

2. Scarborough JE, Ingraham AM, Liepert AE, et al. Nonoperative management is as effective as immediate splenectomy for adult patients with high-grade blunt splenic injury. J Am Coll Surg 2016;223:249-258.

3. Zarzaur BL, Kozar R, Myers JG, et al. The splenic injury outcomes trial. J Trauma Acute Care Surg 2015;79:335-342.

4. Bartlett A, Williams R, Hilton M. Splenic rupture in infectious mononucleosis: A systematic review of published case reports. Injury 2015;47:531-538.

5. Imbert P, Rapp C, Buffet PA. Pathological rupture of the spleen in malaria: Analysis of 55 cases (1958-2008). Travel Med Infect Dis 2009;7:147-159.

6. Oehler RL, Velez AP, Mizrachi M, et al. Bite-related and septic syndromes caused by cats and dogs. Lancet Infect Dis 2009 2009;9:439-447.

7. Butler T. Capnocytophaga canimorsus: An emerging cause of sepsis, meningitis, and post-splenectomy infection after dog bites. Eur J Clin Microbiol Infect Dis 2015;34:1271-1280.

8. Janda JM, Graves MH, Lindquist D, Probert WS. Diagnosing Capnocytophaga canimorsus infections. Emerg Infect Dis. 2006;12:340-342.

9. Brichacek M, Blake P, Kao R. Capnocytophaga canimorsus infection presenting with complete splenic infarction and thrombotic thrombocytopenic purpura: A case report. BMC Res Notes 2012;5:695.

Address correspondence to:

Dr. Louis H. Alarcon

Division of Trauma and General Surgery Department of Surgery University of Pittsburgh Suite F1281 200 Lothrop Street Pittsburgh, PA, USA 15313

E-mail: alarconl@upmc.edu 
Cite this article as: Cyr AR, Zuckerbraun BS, Puyana JC, Alarcon LH (2016) Capnocytophagia sepsis and spontaneous splenic rupture following a minor dog bite. Surgical Infections Case Reports 1:1, 93-96, DOI: $10.1089 /$ crsi.2016.0022
$\mathrm{DVT}=$ deep venous thrombosis

$\mathrm{INR}=$ international normalized ratio 